\title{
Uso de la Simulación Monte Carlo para la Toma de Decisiones en una Línea de Montaje de una Fábrica
}

\author{
Rosangela M. Vanalle, Wagner C. Lucato, Milton Vieira Júnior y Ivone D. Sato \\ Departamento de Ingeniería de Producción, UNINOVE - Universidad Nove de Julho \\ Av. Francisco Matarazzo, 612, 05001-020, São Paulo-Brasil. \\ (e-mail: rvanalle@uninove.br; wlucato@uninove.br; mvieirajr@uninove.br; \\ ivone.sato@uninove.br)
}

Recibido Dic. 16, 2011; Aceptado Ene. 31, 2012; Versión final recibida Feb. 08, 2012

\section{Resumen}

Se presenta una aplicación de la simulación Monte Carlo como una herramienta para la toma de decisiones en una planta fabril. Se ha usado el caso de una línea de montaje de tres estaciones dedicadas a producir termostatos de plancha en una instalación de fabricación de artículos domésticos. Inicialmente la planta fue diseñada para generar una tasa de producción de 3500 montajes por semana, mientras que la producción real solo alcanzaba un $85 \%$ de este valor. Se estudió los tiempos y movimientos y los resultados se utilizaron como una base para la mejora de la operación actual de la línea usando el método de simulación estocástica. Dicha simulación inicial posibilitó la identificación de los problemas claves responsables de la limitada tasa de producción. Varias otras simulaciones fueron llevadas a cabo para probar diferentes mejoras de la línea de montaje.

\section{Monte Carlo Simulation as a Tool for Decision Making in an Assembly Line of a Manufacturing Company}

Monte Carlo simulation is employed as a tool for decision making on a manufacturing company. A three station assembly line dedicated to produce flat iron thermostats in a houseware manufacturing facility is used as a study case. Initially the plant was designed to generate an output of 3500 assemblies per week, while actual production only reached $85 \%$ of that value. Time and movements were studied and the results were used as a basis for improving the existing line operation using the Monte Carlo method. This initial simulation enabled the identification of the key problems responsible for restraining the output. Several other simulations were conducted to test different assembly line arrangements. 


\section{INTRODUCCIÓN}

Actualmente, las empresas se enfrentan a un mercado muy competitivo, lo que ha hecho que tengan que cambiar sus sistemas de gestión para adecuarlos a las nuevas y cambiantes condiciones (Matilla y Chalmeta, 2007). Así, a pesar de que a lo largo de las últimas décadas las estrategias de fabricación han cambiado de la producción en masa a la personalización en masa (Mula et al., 2005), las líneas de montaje continúan siendo ampliamente utilizadas en la industria manufacturera. Una línea es un sistema de fabricación orientado por el flujo de la producción y consistente en sucesivas estaciones de trabajo por lo general dispuestas a lo largo de una cinta transportadora mediante la cual se mueven las piezas de una estación hacia la otra. En cada estación, las operaciones se realizan repetidamente en ciclos de tiempo predeterminados, de tal manera que, al final de la línea, se obtiene un producto intermedio o completamente terminado (Slack et al., 2009; Chase et al. 2006). La revisión de la literatura indica que el tema básico en la investigación de las líneas de montaje ha sido el referente a los problemas de balanceo de línea o Assembly Line Balancing (ALB). Generalmente, un problema de ALB consiste en encontrar un adecuado balanceo de la línea; es decir, la asignación de un determinado número de tareas a una estación en particular observando las actuales relaciones de precedencia y otras restricciones adicionales. Asimismo, los problemas de ALB procuran asignar las tareas a las estaciones de manera que, dado un tiempo ciclo, permita minimizar el número de estaciones requeridas para procesarlas (Boysen et al., 2006). Para explorar los problemas típicos de ALB, han sido empleados varios enfoques: algoritmos genéticos como los propuestos por Ju y Yin (2009) y Levition et al. (2006), técnicas de «colonias de hormigas» (McMullen y Tarasewich, 2003; 2006), modelos de fabricación integrados por computadora (Nkasu y Leung, 1995; Scholl y Klein, 1999), búsqueda de semillas (Erel et al., 2005), ALB orientada al costo (Amen, 2006) y simulación estocástica (Fazlollahtabar, et al., 2010), entre otras (Becker y Scholl, 2006).

Sin embargo, a pesar de que muchos de los problemas analizados en la literatura involucran ejecuciones de las líneas de montaje de primera vez, la mayoría de los problemas de balanceo en las líneas reales se relacionan con sus reconfiguraciones (Falkenauer, 2005; Benítez-Perez, et al., 2009). Esta es la razón fundamental por la que este trabajo propone la utilización de la simulación estocástica, una herramienta que puede utilizarse para identificar dificultades y proponer acciones correctivas para problemas reales observados en la producción de una línea de montaje que ya esté en marcha. Esto se lleva a cabo mediante un estudio de caso de una línea de montaje existente en la que la tasa de producción inicialmente considerada no pudo cumplirse. Como se mostrará a lo largo del trabajo, la ventaja más significativa de la metodología propuesta es la posibilidad de evaluar la tasa de producción real de la línea y probar las modificaciones sugeridas por la simulación de su operación. Esto evita interrupciones en la producción y tiempos de prueba excesivos (costos), los cuales son inevitables si los análisis se efectúan y las modificaciones se comprueban en la línea ya existente en la fábrica. Además, la solución propuesta supondrá una mayor agilidad en la búsqueda de soluciones para los problemas estudiados (Sanchis et al., 2009).

\section{METODOLOGIA}

Hillier y Liberman (1995) sugieren que el primer paso para simular una operación es desarrollar un modelo que represente el sistema a ser analizado. Winston (2003) y Muñoz (2010) también afirman que la forma adecuada para simular una operación como la considerada en este trabajo es a través del desarrollo de un método de simulación de evento discreto utilizando distribuciones de probabilidad conocida como simulación estocástica o método de Monte Carlo. Es relevante añadir que existen otros métodos que también podrían utilizarse para realizar los análisis de los problemas considerados, como la Teoría de las colas. No obstante, la simulación ofrece ciertas ventajas sobre dicha proposición, como podría demostrarse con la utilización del software Simio®, según Kelton et al. (2011).

En el caso de estudios de línea de montaje, puede desarrollarse el siguiente procedimiento de simulación de evento discreto propuesto por Rubinstein y Kroese (2008), y Robert y Casella (2004): 1) Para cada una de las estaciones en una línea de montaje, debe determinarse la distribución de frecuencia de sus tiempos de proceso basándose en medidas de tiempo reales obtenidas por medio de un cronómetro. Entonces, pueden construirse los respectivos diagramas 
de frecuencia relativa acumulativa, como muestra la Figura $1 ; 2)$ Mediante un número aleatorio, el diagrama de la frecuencia relativa acumulativa permite determinar un tiempo de proceso aleatorio para una estación de trabajo en particular (estación $X$ en el ejemplo mostrado más adelante). El procedimiento sugerido funciona como sigue: a) basándose en un generador de números aleatorios, se escoge uno (78 como se muestra en la Figura 1); b) para este número, se asocia la frecuencia acumulativa respectiva (78,3\% en el ejemplo); c) este porcentaje define el primer tiempo de ciclo aleatorio para la estación A (0,65 min/pieza en el ejemplo). Esta simulación podría ampliarse al número deseado de rondas; 3) La línea de montaje entera podría simularse repitiendo el mismo procedimiento para todas las estaciones de trabajo en esa línea y considerando sus operaciones simultáneas, lo cual podría permitir la identificación de eventuales problemas operativos así como la tasa de producción simulada para la línea.

Distribución de la frecuencia - Estatión X
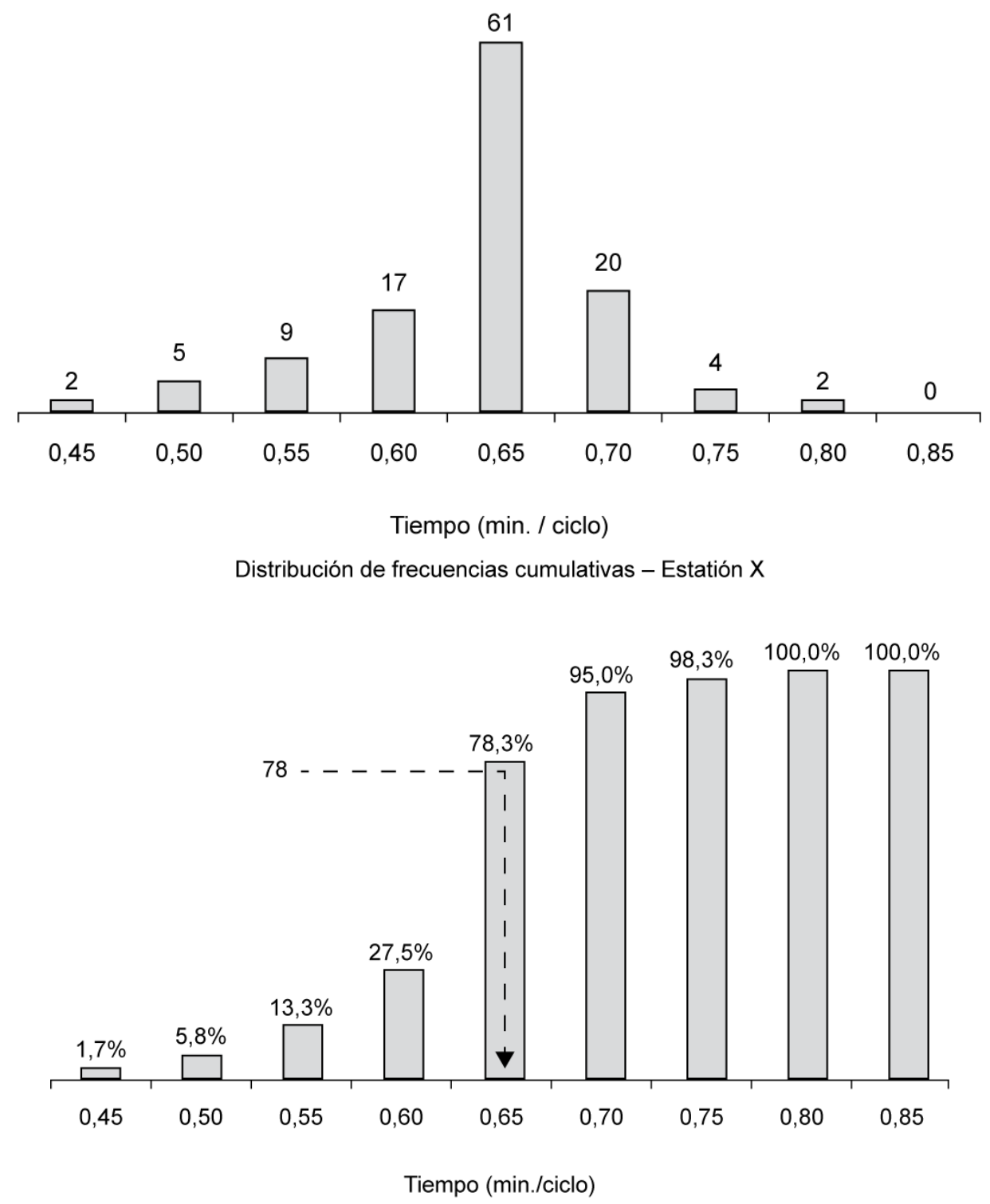

Fig. 1: Procedimiento de la simulación de Monte Carlo

\section{ESTUDIO DE CASO}

El siguiente estudio de caso se presenta como una forma de explorar las posibilidades de usar la simulación estocástica como una herramienta para superar las dificultades de producción. Se trata de una empresa de fabricación de artículos para el hogar localizada en Brasil, subsidiaria de un gran grupo multinacional, que estaba teniendo algunas dificultades de producción con su recién 
instalada línea de montaje para un cierto tipo de termostato de plancha. La compañía había decidido que dicho componente también se fabricase en Brasil, por lo que su Departamento de Ingeniería de Fabricación diseñó una pequeña línea de montaje con tres estaciones de trabajo y un único operario en cada una. Eso debería ser suficiente para generar las 3.500 partes necesarias por semana según los requisitos del momento. El diseño esquemático de la línea se muestra en la Figura 2.

Para poder equilibrar la línea, la Ingeniería de Fabricación recibió de la empresa matriz una lista con las operaciones requeridas para montar el termostato junto con los respectivos tiempos de proceso patrones empleados en el extranjero. Basándose en esta información, se desarrolló un balanceo de la línea de montaje (ALB) dividiendo las operaciones requeridas entre las tres estaciones (Muñoz et al., 2004). Para ese propósito, la Ingeniería de Fabricación decidió agrupar las operaciones que resultarían en el tiempo de proceso más alto entre las tres en la estación de trabajo A, ya que se asumió que la primera estación debería controlar la tasa de producción de la línea. Como resultado, la estación A se diseñó para producir un promedio de 85 piezas por hora (0,706 min/pieza) o 3.740 piezas por semana. Las otras dos estaciones de trabajo fueron diseñadas de manera semejante, y sus tiempos de proceso/tasas de producción también se muestran en la Figura 2. Después de 3 meses en operación, la línea no fue capaz de generar una tasa de producción mayor de 3.200 termostatos por semana durante sus horas de trabajo normal. La cantidad de termostatos requerida para sostener la producción de planchas pudo obtenerse trabajando horas extras, incurriendo como consecuencia en costos excesivos de mano de obra que redujeron drásticamente las ventajas que, supuestamente, tendría la instalación de la línea de montaje en Brasil. Para superar estas dificultades de producción, la empresa brasileña pidió a los investigadores que: a) identificasen las razones claves de por qué la tasa de producción originalmente establecida no podía alcanzarse, y b) sugiriesen modificaciones en el diseño o balanceo de la línea de montaje para poder obtener la requerida tasa de producción de 3.500 piezas por semana trabajando a un ritmo normal durante las horas también normales de trabajo.

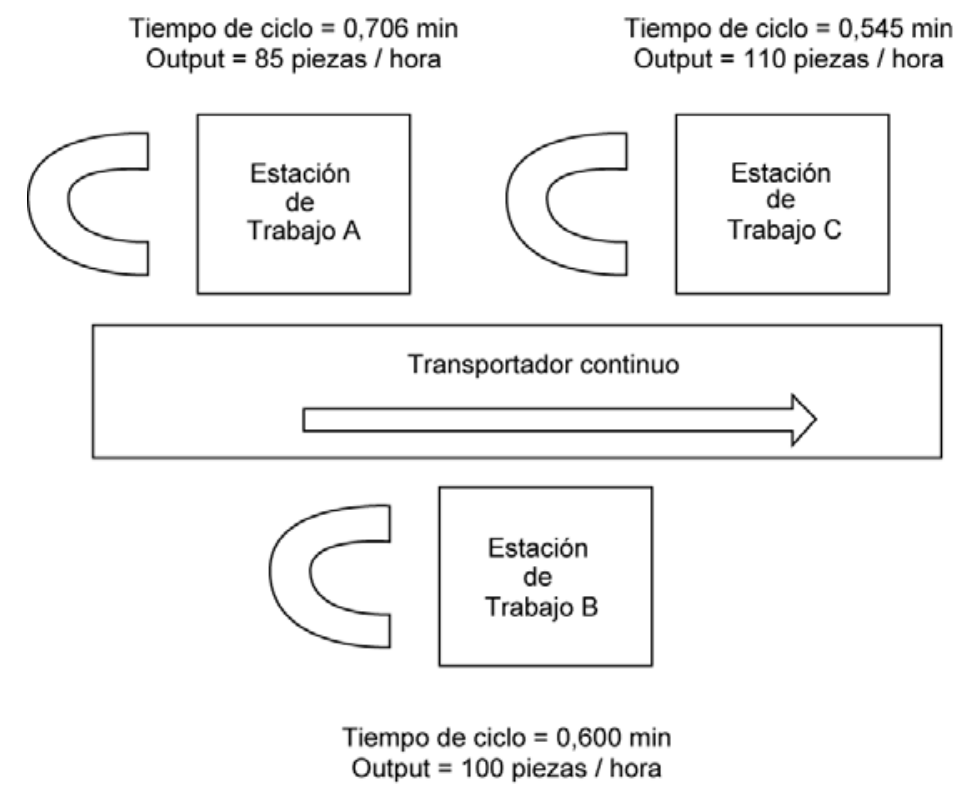

Fig. 2: Diseño de la línea esquemática - Balance inicial

Análisis de la situación actual: para poder identificar posibles problemas con la línea de montaje de termostatos, se determinaron los tiempos de proceso reales para las tres estaciones de la línea manteniendo todo sin alteraciones. Con este propósito, se efectuó la medición de los tiempos con un cronómetro tomando 120 lecturas de cada ciclo para aumentar la precisión y para incorporar eventuales sucesos aleatorios (Barnes, 1999). Los resultados resumidos de las mencionadas tomas de tiempo se muestran en la Tabla 1. 
Tabla 1: Determinaciones actuales de los tiempos de ciclo estándar.

\begin{tabular}{|c|c|c|c|}
\cline { 2 - 4 } \multicolumn{1}{c|}{} & \multicolumn{3}{c|}{ Tiempos de Ciclo (min) } \\
\cline { 2 - 4 } \multicolumn{1}{c|}{} & Estación A & Estación B & Estación C \\
\hline 1 & 0,90 & 0,82 & 0,68 \\
\hline 2 & 0,60 & 0,55 & 0,59 \\
\hline 3 & 0,83 & 0,75 & 0,60 \\
\hline 4 & 0,83 & 0,75 & 0,62 \\
\hline 5 & 0,59 & 0,53 & 0,67 \\
\hline 6 & 0,74 & 0,68 & 0,69 \\
\hline$\ldots \ldots \ldots$ & $\ldots \ldots \ldots$ & $\ldots \ldots \ldots$ & $\ldots \ldots \ldots$ \\
\hline 118 & 0,70 & 0,82 & 0,69 \\
\hline 119 & 0,70 & 0,63 & 0,51 \\
\hline 120 & 0,76 & 0,75 & 0,79 \\
\hline Media & 0,760 & 0,655 & 0,640 \\
\hline S (Desv. Est.) & 0,087 & 0,066 & 0,045 \\
\hline
\end{tabular}

Durante el estudio de la línea de montaje, se tuvo conocimiento de que el equipo de producción no acostumbraba a dejar partes sin montar en la línea para el día siguiente. Como parte de su rutina de trabajo normal, completaban todos los montajes al final del día antes de limpiar sus respectivas estaciones de trabajo, dejando la línea completamente vacía sin ningún componente montado o casi montado. Como parte de este análisis inicial, se hizo una comparación entre los tiempos de proceso considerados en el diseño original de la línea y los tiempos de proceso actuales determinados como parte de esta evaluación (véase la Tabla 2).

Tabla 2: Comparación entre los tiempos de proceso actual y los diseñados.

\begin{tabular}{|c|c|c|c|c|}
\cline { 2 - 5 } \multicolumn{1}{c|}{$(\min /$ parte $)$} & Estación A & Estación B & Estación C & Total \\
\hline Tiempos de proceso diseñados & 0,706 & 0,600 & 0,545 & 1,851 \\
\hline Tiempos de proceso actuales & 0,760 & 0,655 & 0,640 & 2,055 \\
\hline Diferencia $(\%)$ & $+7,6 \%$ & $+9,2 \%$ & $+17,4 \%$ & $+11,0 \%$ \\
\hline
\end{tabular}

Como se puede ver, existe un significativo $11 \%$ de diferencia entre los tiempos de proceso diseñados y los actuales. No obstante, esto no es suficiente para explicar las pérdidas de la producción actual. De ese modo, la estación cuello de botella (bottleneck) de la línea es la estación A con un tiempo de proceso de 0,760 min/pieza. Esto, en principio, supone que podría generar 79 piezas/hora o 3.476 partes por semana, lo que no es compatible con la tasa de producción semanal actual, que tiene 3.200 termostatos como promedio.

Adicionalmente, habría otros problemas aún por identificar. Con el fin de investigar más a fondo otros posibles problemas de la línea de montaje, se decidió simular la operación actual usando la simulación estocástica, ya que las soluciones necesarias para afrontar los problemas de la fábrica necesitan ser rápidas y precisas (Cruz-Machado y Rosa, 2007). Para ilustrar el procedimiento a seguir que se sugiere, está la simulación de los procesos de tiempo para la estación A primeramente basada en los tiempos obtenidos por el cronómetro. Se llevó a cabo la distribución de la frecuencia mostrada en la Figura 3.

El diagrama de la frecuencia relativa acumulativa se preparó como se muestra en la Figura 4. Luego, para poder generar un tiempo de proceso aleatorio para esta estación en particular, se utilizó un generador de números aleatorio y se escogió uno de ellos (76, por ejemplo); b) Para dicho número, tenemos asociada su respectiva frecuencia acumulativa ( $79,2 \%$ en el ejemplo); c) 
Este porcentaje definió el primer tiempo del proceso aleatorio para la estación A $(0,80 \mathrm{~min} / \mathrm{pieza}$ en el ejemplo). Este procedimiento está representado por la línea punteada que se muestra en la Figura 4.

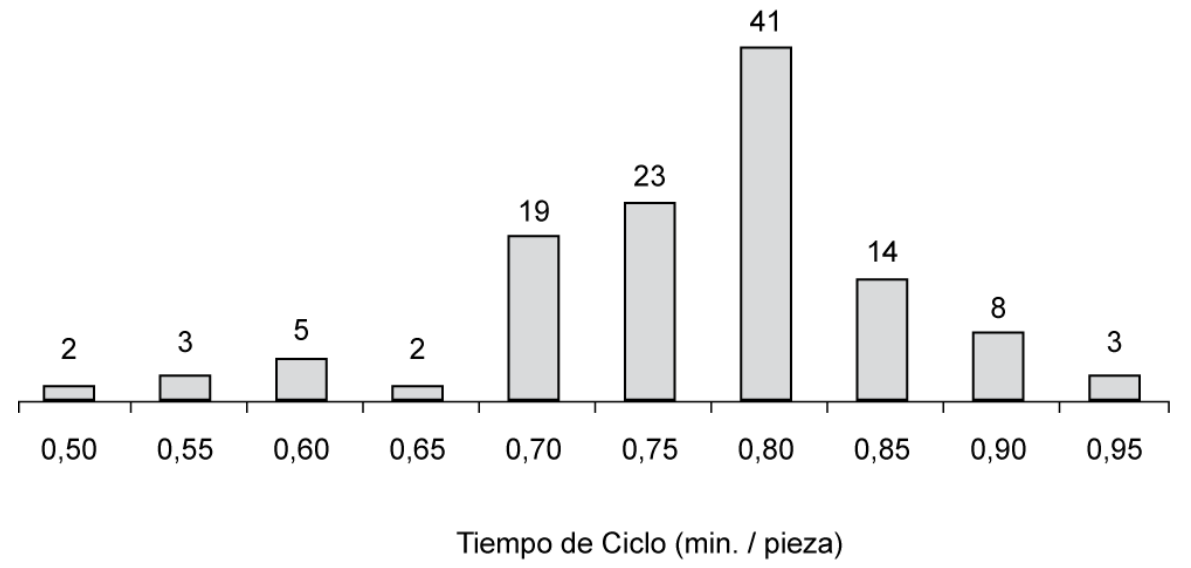

Fig. 3: Distribución de la frecuencia de tiempos de ciclo para la estación A.

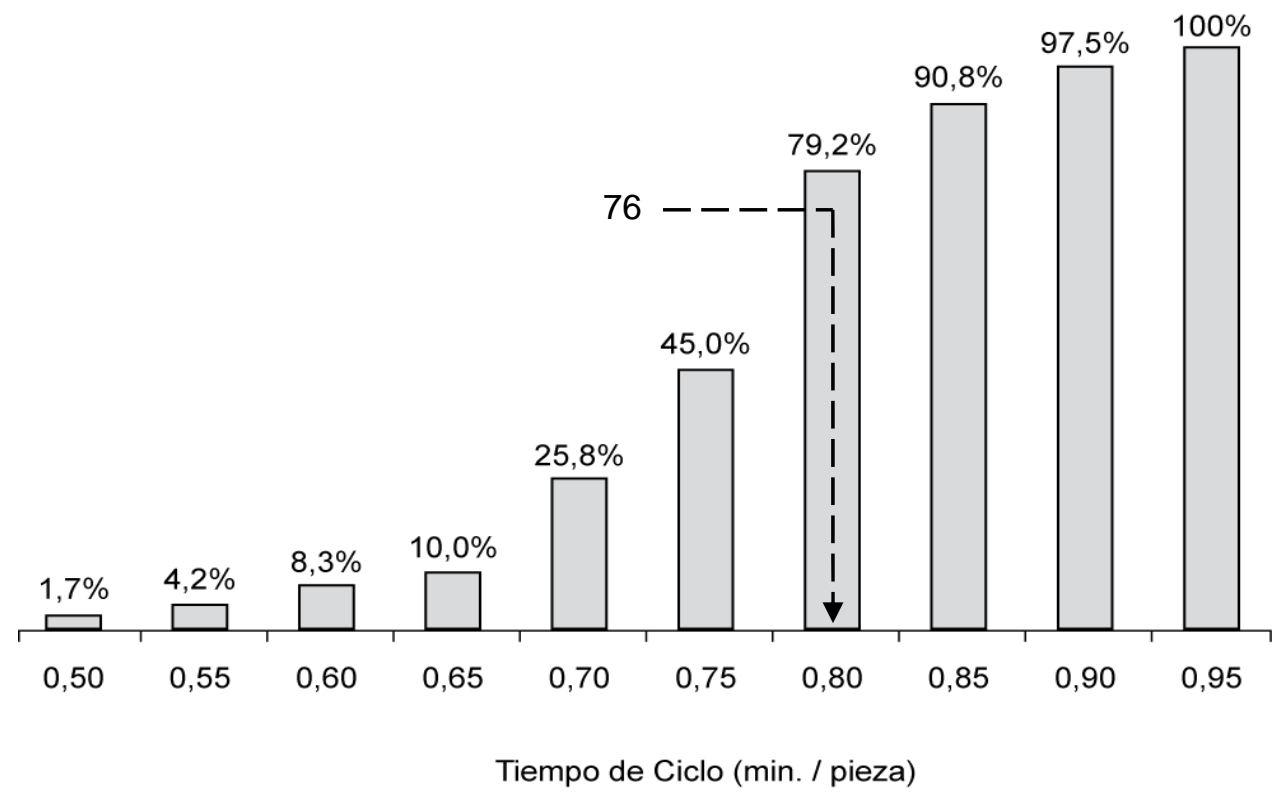

Fig. 4: Diagrama de frecuencia relativa cumulativa para la estación A

Esta misma simulación de tiempo de proceso fue posteriormente repetida hasta 50 veces para la estación A. Considerando que esta estación de trabajo es la primera en la línea, su operación pudo simularse mediante 50 ciclos aleatorios sin la influencia de las operaciones secuenciales. Por supuesto, la suposición fundamental aquí es que ninguna escasez de material afectaría a la operación continua de la estación A. La simulación de 50 procesos se resume en la Tabla 3.

Es interesante tener en cuenta que, de acuerdo con esta simulación, el promedio de tiempo del proceso para la estación A es de 0,772 min, un $2 \%$ más alto que el obtenido durante la medición del tiempo mediante un cronómetro $(0,760 \mathrm{~min})$. Esto es de esperar, ya que la desviación estándar para el proceso de tiempo de la estación A es de 0,085 min. Se llevó a cabo el mismo procedimiento de simulación para las estaciones B y C. Sin embargo, con el fin de simular la operación de la línea completa, se consideró la secuencia de montaje. Por lo tanto, la operación en la estación B solo puede empezar cuando exista una pieza disponible que proceda de la estación A. De la misma manera, la estación C podría empezar su montaje solo si la pieza procedente de la estación B estuviera disponible. La Tabla 4 muestra la completa simulación de la operación de la línea para la situación existente. 
Tabla 3: 50 simulaciones de ciclo para la estación de trabajo A.

\begin{tabular}{|c|c|c|c|c|}
\hline \multirow{3}{*}{ Ciclo } & \multicolumn{4}{|c|}{ Estación A } \\
\hline & \multirow{2}{*}{$\begin{array}{l}\text { Número } \\
\text { Aleatorio }\end{array}$} & \multirow{2}{*}{$\begin{array}{c}\text { Tiempo } \\
\text { de Proceso }\end{array}$} & \multicolumn{2}{|c|}{ Operación } \\
\hline & & & Inicio & Final \\
\hline 1 & 78 & 0,80 & 0,00 & 0,80 \\
\hline 2 & 46 & 0,80 & 0,80 & 1,60 \\
\hline 3 & 68 & 0,80 & 1,60 & 2,40 \\
\hline 4 & 33 & 0,75 & 2,40 & 3,15 \\
\hline 5 & 26 & 0,75 & 3,15 & 3,90 \\
\hline 6 & 96 & 0,90 & 3,90 & 4,80 \\
\hline$\ldots \ldots \ldots$ & ........... & ........... & ............ & ........... \\
\hline 48 & 51 & 0,80 & 36,40 & 37,20 \\
\hline 49 & 6 & 0,60 & 37,20 & 37,80 \\
\hline 50 & 61 & 0,80 & 37,80 & 38,60 \\
\hline & & 38,6 & Promedio & 0,772 \\
\hline
\end{tabular}

Tabla 4: Simulación de la operación de línea completa - Situación existente. (En la Tabla "t" es tiempo de ciclo o de proceso y Oper. es Operario o Operador)

\begin{tabular}{|c|c|c|c|c|c|c|c|c|c|c|c|c|c|c|c|c|}
\hline \multirow{3}{*}{ Ciclo } & \multicolumn{4}{|c|}{ Estación A } & \multicolumn{6}{|c|}{ Estación B } & \multicolumn{6}{|c|}{ Estación C } \\
\hline & \multirow{2}{*}{$\begin{array}{c}\mathrm{Nr} . \\
\text { Rand }\end{array}$} & \multirow{2}{*}{$\mathrm{t}$} & \multicolumn{2}{|c|}{ Reloj } & \multirow{2}{*}{$\begin{array}{l}\text { Nr. } \\
\text { Rand }\end{array}$} & \multirow{2}{*}{$\mathrm{t}$} & \multicolumn{2}{|c|}{ Reloj } & \multirow{2}{*}{$\begin{array}{l}\text { Pieza } \\
\text { Espera }\end{array}$} & \multirow{2}{*}{$\begin{array}{l}\text { Oper. } \\
\text { Espera }\end{array}$} & \multirow{2}{*}{$\begin{array}{c}\mathrm{Nr} . \\
\text { Rand }\end{array}$} & \multirow{2}{*}{$\mathrm{t}$} & \multicolumn{2}{|c|}{ Reloj } & \multirow{2}{*}{$\begin{array}{l}\text { Pieza } \\
\text { Espera }\end{array}$} & \multirow{2}{*}{$\begin{array}{l}\text { Oper. } \\
\text { Espera }\end{array}$} \\
\hline & & & Inicio & Fin & & & Inicio & Fin & & & & & Inicio & Fin & & \\
\hline 1 & 78 & 0.80 & 0.00 & 0.80 & 35 & 0.60 & 0.80 & 1.40 & 0.00 & 0.80 & 15 & 0.60 & 1.40 & 2.00 & 0.00 & 1.40 \\
\hline 2 & 46 & 0.80 & 0.80 & 1.60 & 22 & 0.60 & 1.60 & 2.20 & 0.00 & 0.20 & 99 & 0.80 & 2.20 & 3.00 & 0.00 & 0.20 \\
\hline 3 & 68 & 0.80 & 1.60 & 2.40 & 18 & 0.60 & 2.40 & 3.00 & 0.00 & 0.20 & 80 & 0.70 & 3.00 & 3.70 & 0.00 & 0.00 \\
\hline 4 & 33 & 0.75 & 2.40 & 3.15 & 73 & 0.70 & 3.15 & 3.85 & 0.00 & 0.15 & 67 & 0.65 & 3.85 & 4.50 & 0.00 & 0.15 \\
\hline 5 & 26 & 0.75 & 3.15 & 3.90 & 97 & 0.80 & 3.90 & 4.70 & 0.00 & 0.05 & 74 & 0.65 & 4.70 & 5.35 & 0.00 & 0.20 \\
\hline 6 & 96 & 0.90 & 3.90 & 4.80 & 72 & 0.70 & 4.80 & 5.50 & 0.00 & 0.10 & 87 & 0.70 & 5.50 & 6.20 & 0.00 & 0.15 \\
\hline 7 & 58 & 0.80 & 4.80 & 5.60 & 89 & 0.75 & 5.60 & 6.35 & 0.00 & 0.10 & 74 & 0.65 & 6.35 & 7.00 & 0.00 & 0.15 \\
\hline$\ldots$ & $\cdots$ & $\ldots$ & $\cdots$ & $\cdots$ & $\cdots$ & $\cdots$ & $\cdots$ & $\cdots$ & $\cdots$ & $\cdots$ & $\cdots$ & $\cdots$ & $\cdots$ & $\cdots$ & $\cdots$ & $\ldots$ \\
\hline 48 & 51 & 0.80 & 36.40 & 37.20 & 22 & 0.60 & 37.20 & 37.80 & 0.00 & 0.20 & 78 & 0.80 & 37.80 & 38.60 & 0.00 & 0.10 \\
\hline 49 & 6 & 0.60 & 37.20 & 37.80 & 97 & 0.80 & 37.80 & 38.60 & 0.00 & 0.00 & 85 & 0.80 & 3860 & 39.40 & 0.00 & 0.00 \\
\hline 50 & 61 & 0.80 & 37.80 & 38.60 & 11 & 0.60 & 38.60 & 39.20 & 0.00 & 0.00 & 94 & 0.80 & 39.40 & 40.20 & 0.20 & 0.00 \\
\hline \multicolumn{2}{|c|}{ Totales } & 38.60 & & & & 32.60 & & & 1,15 & 6.60 & & 31.75 & & & 0.70 & 8.15 \\
\hline
\end{tabular}

Para una mejor comprensión, resulta apropiado explicar cómo debe leerse la Tabla 4:

1.- En un determinado día de trabajo, la operación de la línea empieza con la estación de trabajo A realizando su primer montaje. De acuerdo con la simulación, el tiempo del proceso para esta primer parte es de 0,80 min. Mientras tanto, los operarios B y C continúan esperando, ya que la línea se vació el día anterior de acuerdo con las prácticas existentes. Asumiendo la hora cero para el inicio de las operaciones, la estación A termina su primer montaje en 0,8 min. Entonces, empieza inmediatamente su segundo montaje en 0,8 min y lo termina transcurridos 1,60 min -ya que la segunda parte del tiempo del proceso es también de 0,8 min-, y así sucesivamente.

2.- La primera parte montada por la estación A va a la estación B y llega a ella en 0,80 min. El operario, entonces, efectúa su montaje y lo termina en 1,40 (0,60 de tiempo de proceso). Sin embargo, B no puede empezar a trabajar en la segunda parte porque A no la ha liberado para B. De ese modo, el operario de $B$ se queda ocioso durante 0,2 min (véase la columna «Espera del operario»). En un tiempo de reloj de $1,60 \mathrm{~min}$, B empieza a montarla tras haber recibido la segunda parte de $A$, lo que se completa después de 2,20 min (0,60 de tiempo de proceso). 
3.- Lo mismo ocurre en la estación C. Aquí, el operario permanece ocioso durante 1,40 min hasta que la primera parte llega a su posición. En ese momento, se realiza el montaje en la estación C y termina en un tiempo de reloj de 2,00 min (también 0,60 tiempo del proceso). Como la segunda parte solo será liberada para B a los 2,20 min, el operario de C también se quedará ocioso durante $0,20 \mathrm{~min}$ adicionales.

4.- Siguiendo el mismo procedimiento anteriormente expuesto, puede entenderse la operación de la línea completa para la simulación entera de 50 ciclos.

Tras analizar los resultados obtenidos en la simulación de la línea de montaje, se puede llegar a las siguientes conclusiones:

1.- El operario de A trabaja continuamente y sin interrupción alguna para alimentar a la estación B con montajes.

2.- Durante la simulación de 50 procesos, el operario de $B$ permanece ocioso durante 6,60 min esperando las partes que serán liberadas por la estación A. Esto representa una pérdida de trabajo del $17 \%$ en relación con el tiempo que el trabajador permanece en el lugar de trabajo $(39,20 \mathrm{~min})$.

3.- Del mismo modo, el operario de $C$ permanece ocioso durante 8,15 min o un $20 \%$ de sus 40,20 min de tiempo de trabajo;

4.- Los 50 montajes considerados en la simulación se completaron en 40,20 min (momento en que el operario de $\mathrm{C}$ termina la 50 . $^{\mathrm{a}}$ pieza), lo que corresponde a un promedio de la tasa de producción de la línea de una pieza cada 0,804 min o de 74 piezas por hora.

5.- Es interesante tener en cuenta que las 74 piezas por hora generarían 3.256 partes por semana, cifra muy próxima a la tasa de producción actual de la línea (cerca de 3.200 montajes semanales).

Como resultado de la simulación, fue posible identificar los problemas claves que afectan al desempeño de la línea actual: a) los tiempos de proceso estándar actuales son un 11\% mayores que los originalmente diseñados, pero esta diferencia no afecta significativamente a la tasa de producción necesaria (3.476 actual frente a 3.500 objetivo); b) la práctica de vaciar la línea al final del día genera un retraso diario de 1,40 min, lo que significa la pérdida de más de 200 piezas por semana; c) como resultado del balanceo de la línea de montaje actual (ALB), las variaciones estadísticas de los tiempos de proceso generan ociosidad en los operarios de las estaciones B y C, lo que supone el resto de la reducción observada en la tasa de producción.

Mejoras sugeridas: basándose en los resultados obtenidos durante la simulación del estado actual, se llevaron a cabo algunos ajustes en el balanceo y en las prácticas de la línea. El punto más importante que debe enfatizarse es que las modificaciones no fueron comprobadas en la fábrica, pues esto probablemente habría supuesto interrumpir la producción aún más, incurriendo en un significativo tiempo de implementación y de costos extraordinarios. Por este motivo, dichas modificaciones se evaluaron mediante simulaciones adicionales. De esta manera, solamente podría implementarse la alternativa recomendada final minimizando los recursos necesarios para desarrollar la solución optimizada. Como resultado de varias simulaciones alternativas, los siguientes cambios fueron finalmente considerados: a) Modificar la actual rutina de la planta consistente en dejar la línea vacía al final del día. Se consultó a los departamentos de Ingeniería y de Calidad sobre este aspecto, y estos indicaron que no pondrían objeción alguna a la práctica consistente en dejar las piezas inacabadas en las estaciones de trabajo hasta el día siguiente. La decisión de hacer este cambio fue, entonces, adoptada para la simulación. b) Las operaciones fueron rearregladas entre las tres estaciones dejando a la estación $\mathrm{C}$ con el proceso de tiempo más alto, como se muestra en la Tabla 5. 
Tabla 5: Redistribución de los ciclos de tiempo

\begin{tabular}{|c|c|c|c|c|}
\cline { 2 - 5 } \multicolumn{1}{c|}{$(\mathrm{min} /$ pieza $)$} & Estación A & Estación B & Estación C & Total \\
\hline Tiempos de proceso originales & 0,760 & 0,655 & 0,640 & 2,055 \\
\hline Tiempo de proceso revisados & 0,675 & 0,685 & 0,695 & 2,055 \\
\hline
\end{tabular}

Ya que las estaciones $A$ y $B$ tienen ahora tiempos de proceso más bajos que la estación $C$, un número creciente de WIP (Work in Process) se acumularía después de A y B si sus respectivos operarios trabajaran continuamente. Por lo tanto, se estableció un número máximo de cinco montajes entre las estaciones, de tal manera que si, por cualquier motivo, un operario identificase cinco montajes esperando a ser procesados por la siguiente estación, pararía para evitar un excesivo aumento de WIP. Con el objeto de poder comprobar la adecuación de las modificaciones propuestas, se realizó una nueva simulación como se muestra de forma resumida en la Tabla 6.

Tabla 6: Simulación de operación de la línea completa después de los cambios

\begin{tabular}{|c|c|c|c|c|c|c|c|c|c|c|c|c|c|c|c|c|}
\hline \multirow{3}{*}{ Ciclo } & \multicolumn{4}{|c|}{ Estación A } & \multicolumn{6}{|c|}{ Estación B } & \multicolumn{6}{|c|}{ Estación C } \\
\hline & \multirow{2}{*}{$\begin{array}{c}\text { Nr. } \\
\text { Rand }\end{array}$} & \multirow{2}{*}{$\begin{array}{c}\text { Tiempo } \\
\text { de } \\
\text { ciclo }\end{array}$} & \multicolumn{2}{|c|}{ Reloj } & \multirow{2}{*}{$\begin{array}{l}\mathrm{Nr} . \\
\text { Rand }\end{array}$} & \multirow{2}{*}{$\begin{array}{c}\text { Tiempo } \\
\text { de } \\
\text { ciclo } \\
\end{array}$} & \multicolumn{2}{|c|}{ Reloj } & \multirow{2}{*}{$\begin{array}{l}\text { Pieza } \\
\text { Espera }\end{array}$} & \multirow{2}{*}{$\begin{array}{c}\text { Operador } \\
\text { Espera }\end{array}$} & \multirow{2}{*}{$\begin{array}{c}\text { Nr. } \\
\text { Rand }\end{array}$} & \multirow{2}{*}{$\begin{array}{c}\text { Tiempo } \\
\text { de } \\
\text { ciclo } \\
\end{array}$} & \multicolumn{2}{|c|}{ Reloj } & \multirow{2}{*}{$\begin{array}{l}\text { Pieza } \\
\text { Espera }\end{array}$} & \multirow{2}{*}{$\begin{array}{c}\text { Operador } \\
\text { Espera }\end{array}$} \\
\hline & & & Inicio & Fin & & & Inicio & Fin & & & & & Inicio & Fin & & \\
\hline 1 & 15 & 0.64 & 0.00 & 0.64 & 35 & 0.64 & 0.00 & 0.64 & 0.00 & & 78 & 0.73 & & 0.73 & & \\
\hline 2 & 99 & 0.84 & 0.64 & 1.48 & 22 & 0.64 & 0.64 & 1.28 & 0.00 & & 46 & 0.73 & 0.80 & 1.53 & & \\
\hline 3 & 80 & 0.74 & 1.48 & 2.22 & 18 & 0.64 & $1-48$ & 2.12 & 0.00 & 0,2 & 68 & 0.73 & 1.53 & 2.26 & & \\
\hline 4 & 67 & 0.69 & 2.22 & 2.91 & 73 & 0.74 & 2.22 & 2.96 & 0.00 & 0,1 & 33 & 0.68 & 2.26 & 2.94 & & \\
\hline 5 & 74 & 0.69 & 2.91 & 3.60 & 97 & 0.78 & 2.94 & 3.72 & 0.03 & & 26 & 0.68 & 2.96 & 3.64 & & 0.02 \\
\hline 6 & 87 & 0.74 & 3.60 & 4.34 & 72 & 0.74 & 3.78 & 4.52 & & & & & 3.72 & 4.55 & & 0.08 \\
\hline 48 & 78 & 0.69 & 31.68 & 32.37 & 22 & 0.64 & 32.76 & 33.40 & 1.08 & & 51 & 0.73 & 33.31 & 34.04 & 0.55 & \\
\hline 49 & 49 & 0.69 & 32.37 & 33.06 & 97 & 0.84 & 33.40 & 34.24 & 1.03 & & 6 & 0.53 & 34.04 & 34.57 & 0.64 & \\
\hline 50 & 34 & 0.69 & 33.06 & 33.75 & 11 & 0.64 & 34.24 & 34.88 & 1.18 & & 61 & 0.73 & 34.57 & 35.30 & 0.33 & \\
\hline \multicolumn{2}{|c|}{ Totais } & 33.75 & & & & 34.54 & & & 44.93 & 0.30 & & 35.10 & & & 26.80 & 0.13 \\
\hline
\end{tabular}

Algunas mejorías pudieron identificarse en esta nueva simulación. Inicialmente, la línea se dejaba debidamente alimentada con montajes inacabados en cada estación de trabajo. Al principio del día, los operarios podían empezar su trabajo inmediatamente, eliminando la pereza inherente a la práctica habitual de dejar la línea vacía al final del día. Las estaciones A y B también tienen ahora una tasa de producción mayor que la $\mathrm{C}$, lo que prácticamente elimina toda ociosidad del operario. Manteniendo el WIP limitado a 5 partes entre las estaciones, se evita el almacenamiento excesivo de piezas entre las mismas. Asimismo, el nuevo balanceo de la línea posibilitó el montaje de 50 piezas en 35,30 minutos (momento en que el operario $C$ termina la $50^{a}$ pieza), lo que supone un promedio de tiempo de proceso de la línea de montaje de 0,706 min/pieza, 85 piezas por hora 0 3.740 termostatos por semana. Es importante mencionar que el hecho de que la simulación produjera exactamente el mismo promedio de tiempo de proceso que la estación A en la situación actual fue pura coincidencia, ya que no existe relación alguna entre ellas. Como pudo verse, los cambios propuestos en las prácticas de fabricación y en el balanceado de la línea fueron capaces de eliminar las actuales limitaciones de manufactura, permitiendo que el volumen de producción alcanzase el rendimiento deseado en la tasa de producción. Estas modificaciones han sido recomendadas para su implementación. 


\section{CONCLUSIONES}

En la planta fabril, las soluciones bien diseñadas no siempre se convierten en una realidad práctica, como el caso presentado pudo demostrar. Algunos problemas no anticipados por los diseñadores de la línea de montaje afectaron a la tasa de producción, lo que requirió horas extraordinarias de trabajo (y costo adicional) para superar esa dificultad. Este trabajo presenta un abordaje diferente para identificar y analizar los cambios propuestos por medio de la técnica de simulación estocástica.

El punto clave del presente artículo, que se demostró con el desarrollo de este trabajo, es la posibilidad de ejecutar y probar diversas alternativas de mejora de una línea de montaje en un entorno virtual. El aspecto más relevante es que la solución propuesta es muy innovadora, ya que aporta un flujo de nuevos conocimientos, competencias y capacidades sin interrumpir la producción y con costos mínimos (Espinosa et al., 2008). Se trata, así, de un procedimiento de ingeniería que podrá suponer beneficios evidentes para quienes trabajan en la fábrica.

Finalmente, como confirmación de la posibilidad de aplicar de una forma práctica el desarrollo propuesto, es importante mencionar que los cambios sugeridos en este trabajo fueron finalmente adoptados por la empresa de fabricación de artículos domésticos objeto del estudio, y que los rendimientos de la producción alcanzaron los niveles deseados después de un par de días de entrenamiento. En definitiva, pudo solucionarse un incómodo problema de producción mediante la utilización de la simulación estocástica, con una interrupción mínima de dicha producción y con costos insignificantes.

\section{AGRADECIMIENTOS}

Los autores desean expresar su agradecimiento al Fundo de Apoyo a la Pesquisa de la Universidad Nove de Julho (FAP / UNINOVE).

\section{REFERENCIAS}

Amen, M., Cost-oriented assembly line balancing model formulations, solution defficulties, upper and lower bounds. European Journal of Operations Research, Vol, 168, pp, 747-770 (2006).

Barnes, R.M., Estudo de movimentos e de tempos: projeto e medida do trabalho. Edgard Blücher, São Paulo, (1999).

Becker, C. y Scholl, A., A survey on problems and methods in generalized assembly line balancing. European Journal of Operations Research, Vol, 118, pp, 694-715, (2006).

Benítez-Pérez, H.; Cárdenas-Flores, F. y García-Nocetti, F., Red reconfigurable mediante el modelo de control predictivo para tres bandas transportadoras como caso de estudio. Información Tecnológica, Vol.20, No.1, pp.111-127 (2009).

Boysen, N.; Fliedner, M. y Scholl, A., Assembly line Balancing: Which model to use when? Arbeitsund Diskussionspapiere der Wirtschaftswissenschaftlichen Fakultät der Friedrich-SchillerUniversität Jena, (2006).

Chase, R.B.; Jacobs, F.R. y Aquilano, N.J., Administração da produção para a vantagem competitiva, 10.ed., Bookman, Porto Alegre, (2006).

Cruz-Machado, V. y Rosa, P., Modelo de planificación baseado em construcción ajustada para obras de corta duración. Información Tecnológica, Vol.18, No.1, pp.107-118 (2007).

Erel, E.; Subuncuoglu, I. y Sekerci, H., Stochastic assembly line balancing using bean search. International Journal of Production Research, Vol, 43, pp, 1411-1426, (2005).

Espinosa, F.F., Dias, A. y Back, N. Un procedimiento de evaluación de las condiciones necesarias para innovar la gestión de mantenimiento en una empresa. Información Tecnológica, Vol.19, No.1, pp.97-104 (2008). 
Falkenauer, E. Line balancing in the real world, In: Proceedings of the International Conference on Product Lifecycle Management PLM 05, (on cd-rom), Lumiere University of Lyon, France, (2005).

Fazlollahtabar, H.; Hajmohammadi, H. y Es'haghzadeh, A., A heuristic methodology for assembly line balancing considering stochastic time and validity testing. International Journal of Advanced Manufacturing Technology, (2010).

Hillier, F.S. y Lieberman, G. J., Introduction to operations research, McGraw Hill, New York, NY, (1995).

Ju, J. y Yin, Y., Assembly line balancing based on an adaptive genetic algorithm. International Journal of Advanced Manufacturing Technology, Vol, 48, pp. 347-354, (2009).

Kelton, W.D., Smith, J.S., Sturrock D.T. y. Muñoz D.F., Simio y Simulación, Modelado, Análisis, Aplicaciones, Simio LLC, edición e-book en español disponible en http://www.simio.com/publications/SASMAA/, (2011).

Levition, G.; Rubinovitz, J. y Shnits, B., A genetic algorithm for robotic assembly line balancing. European Journal of Operations Research, Vol, 168, pp, 811-825, (2006).

Matilla, M.M. y Chalmeta, R. Metodología para la implantación de unsistema de medición del rendimiento empresarial. Información Tecnológica, Vol.18, No.1, pp.119-126 (2007).

Mcmullen, P.R. y Tarasewich, P., Using ant colony technique to solve the assembly line balancing problem. IIE Transactions, Vol. 35, pp. 605-617, (2003).

Mcmullen, P.R. y Tarasewich, P., Multi-objective assembly line balancing via a modified ant colony optimization technique. International Journal of Production Research, Vol. 44, No,1, pp. 27-34, (2006).

Mula, J., Poler, R. y Garcia, J.P., Evaluación de sistemas para la planificación y control de la producción. Información Tecnológica, Vol.17, No.1, pp.19-34 (2005).

Muñoz, E.; Rosado P. y González F., Modelado de información para la caracterización de utillaje en un sistema de planificación de procesos asistido por computador. Información Tecnológica, Vol. 15, N4, pp. 9-14, (2004).

Muñoz, D.F., Planeación y control de proyectos con diferentes tipos de precedencias utilizando simulación estocástica. Información Tecnológica, Vol. 21, No.4, pp. 25-33, (2010).

Nkasu, M.M. y Leung, K.H., Computer-integrated manufacturing assembly system design. Integrated Manufacturing Systems, Vol. 6 No. 6, pp. 4-14, (1995).

Robert, C.P. y Casella, G., Monte Carlo statistical methods. Springer-Verlag, London, (2004).

Rubinstein, R.Y. y Kroese, D.P., Simulation and the Monte Carlo method. Wiley, Hoboken, NJ, (2008).

Sanchis, R., Poler, R. y Ortiz, A., Técnicas para el modelado de proceso de negocio en cadenas de suministro. Información Tecnológica, Vol. 20, №2, pp. 29-40, (2009).

Scholl, A. y Klein, R., Balancing assembly line effectively - a computerized comparison. European Journal of Operations Research, Vol. 14, pp., 50-58, (1999).

Slack, N.; Chambers, S. y Johnston, R., Administração da produção, 3.ed., Atlas, São Paulo, (2009).

Winston, W. L. Operations research - applications and algorithms. Duxbury Press, Belmont, CA, (2003). 
